\title{
Das Schweizerische Medizin-Forum: an der Praxis orientiert, dem Föderalismus verpflichtet
}
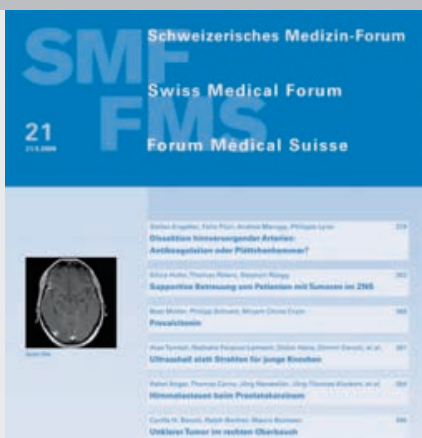

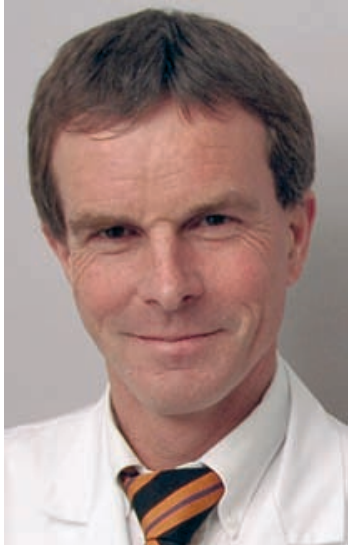

Reto Krapf
Die Neubesetzung und die Erweiterung des Redaktionsteams des Schweizerischen MedizinForums (siehe Artikel Seite 979) durch einen zusätzlichen Vertreter aus der Romandie, Professor Dr. G. Waeber, Lausanne, und einen Vertreter der Hausarztmedizin, Professor P. Tschudi, Basel, illustrieren zwei ganz wichtige Anliegen der Redaktion des Schweizerischen Medizin-Forums: Wir wollen einerseits qualitativ hochstehende und vor allem unabhängige Fort- und Weiterbildung für die niedergelassenen und in Ausbildung stehenden Kolleginnen und Kollegen anbieten. Durch Einbezug von Redaktoren und Reviewern, die als niedergelassene Ärzte tätig sind, hoffen wir, die Praxisrelevanz immer ganz speziell zu gewährleisten und zu fördern. Um möglichst viele Ärztinnen und Ärzte in unserem Lande zu erreichen ist, andererseits, dazu mindestens die Zweisprachigkeit der Zeitschrift von entscheidender Bedeutung, und wir freuen uns, dass nun drei Kollegen aus der Romandie als wichtige Stützen im Redaktionsteam mitarbeiten und der Verlag sich nicht scheut, die erheblichen Aufwendungen für die Übersetzungen zu tätigen.

Das Privileg eines Gastbeitrags in unserer «Schwesterzeitschrift» möchte ich vorwiegend als Anlass nehmen zu danken. Wir sind allen unseren Leserinnen und Lesern für die offensichtliche Anerkennung und Wertschätzung des Schweizerischen Medizin-Forums - sei dies im Rahmen von Leserumfragen dokumentiert, sei dies durch spontane Äusserungen mitgeteilt sehr dankbar. Diese Wertschätzung möchten wir vor allem unseren vielen Autorinnen und Autoren weitergeben, die in nach wie vor überwältigender Weise unseren Anfragen nach Manuskripten fast immer nachkommen. Es freut uns auch, dass immer mehr Kolleginnen und Kollegen spontane Einreichungen vornehmen. Wir bemühen uns, den Autorinnen und Autoren als Gegenleistung qualitativ gute, unabhängige interne und externe Reviews der Manuskripte zu bieten, und bemerken mit Freude die Tatsache, dass sich die Autorinnen und Autoren in unserer Zeitschrift einer grossen Wahrnehmung und Sicht- barkeit sicher sein dürfen. Unser Dank gilt natürlich auch unseren Reviewern, die ihre Aufgabe hautpsächlich in einer Hilfegebung für die Autoren und einer Verbesserung der Inhalte und der Lesbarkeit sehen. Sie heben sich so wohltuend vom Verhalten bei anderen Fachzeitschriften ab, wo es etwa Reviewer geben kann, die als allwissende Schiedsrichter aufzutreten pflegen.

Wir wollen uns auf diesen kleinen Lorbeeren aber nicht ausruhen, sondern ich freue mich, mit dem neuen Redaktionsteam die Zeitschrift weiterzuentwickeln und möglichst noch besser den Bedürfnissen unserer Leserinnen und Leser anzupassen. Wir sind froh, dass die tatkräftige und nachhaltige Unterstützung durch den Verlag und die wohlwollende Förderung durch den EMH-Verwaltungsrat einen günstigen Boden für diese Entwicklung darstellt. Wir sind so überzeugt, dass wir auch unter etwas erschwerteren Bedingungen (z. B. unsicherere Finanzierung durch verändertes Inserentenverhalten) dieses Ziel kontinuierlich verfolgen und erreichen werden. Dafür sind aber ein Dialog mit der und Kritik durch die Leserschaft ganz wichtige Bedingungen. Rufen Sie uns an oder schreiben Sie uns einfach! (E-Mail: smf@emh.ch, Internet: www.medicalforum.ch.) Ideen zu Artikeln, Vorschläge für Autoren, Wünsche an bestehende oder neue Rubriken, auch Kritik, sei sie im Rahmen eines Leserbriefes oder auch formlos geäussert, sind uns sehr willkommen. Wir laden auch die verschiedenen Fachgesellschaften ein, uns ihre Vorstellungen und Bedürfnisse mitzuteilen, und freuen uns, wenn die bisherige angenehme Zusammenarbeit in $\mathrm{Zu}$ kunft noch weiter ausgebaut werden könnte.

Mit diesem Aufruf begrüsse ich die beiden neuen Kollegen ganz herzlich in der Redaktion des Schweizerischen Medizin-Forums. Der neue Schwung wird zusammen mit Ihnen, liebe Leserinnen und Leser, Gewähr bieten, die Zeitschrift weiter zu verbessern, auf ihre Praxisrelevanz zu prüfen und für Kolleginnen und Kollegen in der ganzen Schweiz aktuell zu gestalten.

Reto Krapf* 